\title{
EDITORIAL
}

\section{New editorial policies: content and electronic submission}

Molecular Psychiatry is now entering its seventh year. Many changes are occurring in our field, which are reflected in the journal, both in terms of content and format.

To begin with we would like to thank authors and readers for having made such significant contributions to our field. Our impact factor of 8.93 (2nd in psychiatry and 9th in neuroscience) reflects the excitement and growth of the field of molecular psychiatry and the fact that our journal, which has fostered the field since its infancy, is now the leading journal for reporting breakthroughs in molecular psychiatry.

Our content will continue to reflect our initial editorial goal of having an optimal balance of neuroscience, imaging, genetics and genomics, and patient-oriented investigation. We have discussed with members of the editorial board and experts in the field guidelines for publication. The short answer to our inquiries is that there are no easy formulas and therefore no rigid guidelines. Editorial decisions will be based on common sense and scientific merit.

A particularly difficult editorial challenge is presented by the high number of genetic association studies that has emerged in recent years. After much thought and consideration I do not feel comfortable to prescribe rigid criteria for publication of that type of study. Such criteria might exclude important discoveries. For example, Allen Rose's landmark report of increased frequency of apolipoprotein E type 4 allele in (late-onset familial) Alzheimer's disease had 30 cases and 91 unrelated controls (PNAS 1993; 90: 19771978). Therefore, we will continue to be flexible but rigorous. Strong and compelling justification should be provided both in the cover letter and in the body of the paper. As almost any gene is of 'potential' relevance to psychiatry, before we consider an association study for publication, editor and reviewers will rigorously examine issues such as: Is this study of real value? Why these genes? Why these polymorphisms? Are they coding or non-coding, functional or non-functional? Why this $n$ ? Why this sample? What is the effect size? How much other data have been submitted from this sample? Cover letters and articles must address these questions directly and compellingly.

Once a sample is collected for a genetic study it can now be tested repeatedly, potentially resulting in multiple submissions. Before doing that, authors ought to remember that editors and reviewers will be asking the question 'How many publications can one extract from a single sample set?' For obvious reasons, we strongly discourage papers that report only the testing of one more SNP, particularly in a previously reported sample. We will now only consider articles that provide a truly compelling scientific rationale for genetic associ- ation, and that describe methods, sample, and sample size following generally accepted guidelines and leading to conclusive results. We require that authors strongly justify why a previously reported sample (or an expanded sample) needs to be the object of a new article. Articles submitted to Molecular Psychiatry must contain a description and discussion of all previous and concurrent findings that emerged from a particular sample. It is also crucial that effect size and estimated sample size needed to either confirm or disprove that effect are presented and discussed. We also encourage authors of association studies to consider our Scientific Correspondence format.

In terms of the submission process, editors and publishers alike are becoming increasingly aware of the opportunities electronic submission and peer review offer their communities. We have therefore made electronic submissions available, and strongly encourage the use of our website (http://www.mp-submit.org) for any new submissions and resubmissions.

Our submission website uses a system called 'eJournalPress,' which is a completely web-based tool designed for manuscript submission, peer review, and tracking. It requires no special software to use the system and is easy to use simply following the instructions provided on the website.

It is important that we point out the key benefits of this new system. For authors there is ease and flexibility of submission, faster turnaround by use of the internet for transmitting information that previously relied on either courier or the postal service, and the ability to track the progress of their manuscript through the peer review process. For reviewers, this new system offers a reliable webbased system for transmitting information.

More information about electronic submissions can be found by going to the journal's home page (http://www.nature.com/mp) and reading about submitting online. It is important to stress that although we prefer submissions to be made online, authors can still submit in print to the Editorial Office. Please read our notes for contributors before submitting your article (http://www.naturesj.com/mp/instructions.html).

We thank our authors and readers for the continued support given to Molecular Psychiatry. As we start our seventh volume, our publication frequency and page number will increase so that we can publish a higher number of papers and decrease the time from submission to publication. This will permit us to continue to offer rigorous review, high visibility, and rapid publication.

J Licinio, MD

UCLA Laboratory of Pharmacogenomics E-mail: licinio@ucla.edu 